\title{
ON THE EXTENSION OF ISOMETRIES BETWEEN THE UNIT SPHERES OF A C*-ALGEBRA AND $B(H)$
}

\author{
FRANCISCO J. FERNÁNDEZ-POLO AND ANTONIO M. PERALTA \\ Dedicated to the memory of Professor Joseph Diestel
}

\begin{abstract}
Given two complex Hilbert spaces $H$ and $K$, let $S(B(H))$ and $S(B(K))$ denote the unit spheres of the $\mathrm{C}^{*}$-algebras $B(H)$ and $B(K)$ of all bounded linear operators on $H$ and $K$, respectively. We prove that every surjective isometry $f: S(B(K)) \rightarrow S(B(H))$ admits an extension to a surjective complex linear or conjugate linear isometry $T: B(K) \rightarrow B(H)$. This provides a positive answer to Tingley's problem in the setting of $B(H)$ spaces.
\end{abstract}

\section{INTRODUCTION}

Let $X$ and $Y$ be normed spaces, whose unit spheres are denoted by $S(X)$ and $S(Y)$, respectively. Suppose $T: X \rightarrow Y$ is a surjective real linear isometry. The restriction $\left.T\right|_{S(X)}: S(X) \rightarrow S(Y)$ defines a surjective isometry. The so-called Tingley's problem, named after the contribution of D. Tingley [36], asks if every surjective isometry $f: S(X) \rightarrow S(Y)$ arises in this way, or equivalently, if every surjective isometry $f: S(X) \rightarrow S(Y)$ admits an extension to a surjective real linear isometry $T: X \rightarrow Y$. Tingley's achievements show that, for finite dimensional normed spaces $X$ and $Y$, every surjective isometry $f: S(X) \rightarrow S(Y)$ satisfies $f(-x)=-f(x)$ for every $x \in S(X)$ (see [36, THEOREM on page 377]).

A solution to Tingley's problem has been pursued by many researchers during the last thirty years. Positive answers to Tingley's problem have been established for $\ell^{p}(\Gamma)$ spaces with $1 \leq p \leq \infty$ (see [7,8,10 and [1]), $L^{p}(\Omega, \Sigma, \mu)$ spaces, where $(\Omega, \Sigma, \mu)$ is a $\sigma$-finite measure space and $1 \leq p \leq \infty$ (compare [29, 30] and [31]), and $C_{0}(L)$ spaces (see [37]). Tingley's problem also admits a positive solution in the case of finite dimensional polyhedral Banach spaces (see 21]). The reader is referred to the surveys [12] and [38] for additional details.

In the non-commutative setting, Tingley's problem has been solved for surjective isometries between the unit spheres of two finite dimensional $\mathrm{C}^{*}$-algebras (see [34]) and for surjective isometries between the unit spheres of two finite von Neumann algebras [35]. A more recent contribution solves Tingley's problem for surjective isometries between the unit spheres of spaces, $K(H)$, of compact linear operators on a complex Hilbert space $H$, or more generally, for surjective isometries between the unit spheres of two compact $\mathrm{C}^{*}$-algebras [25, Theorem 3.14]. The novelties in 25] are based on the application of techniques of $\mathrm{JB}^{*}$-triples, and in the abovereferenced paper Tingley's problem is also solved for surjective isometries between

Received by the editors May 5, 2017, and, in revised form, July 4, 2017 and August 1, 2017.

2010 Mathematics Subject Classification. Primary 47B49; Secondary 46A22, 46B20, 46B04, 46A16, 46E40.

Key words and phrases. Tingley's problem, extension of isometries, $\mathrm{C}^{*}$-algebras, $B(H)$. 
the unit spheres of two weakly compact $\mathrm{JB}^{*}$-triples of rank greater than or equal to 5. In [17] we establish a complete solution to Tingley's problem for arbitrary weakly compact $\mathrm{JB}^{*}$-triples. A solution to Tingley's problem for spaces of trace class operators is presented in [18.

Tingley's problem for surjective isometries between the unit spheres of two $B(H)$ spaces seems to be the last frontier in the studies on Tingley's problem. This paper is devoted to providing a complete solution in this case.

The results in 17, 25, 34 are based, among other techniques, on those theorems describing the (maximal) norm closed proper faces of the closed unit ball of a $\mathrm{C}^{*}$ algebra (see [2]) or of a JB*-triple (see [13]). Throughout the paper, the closed unit ball of a normed space $X$ will be denoted by $\mathcal{B}_{X}$. It is shown in [17, 25, 34] that, for a compact $\mathrm{C}^{*}$-algebra $A$ (respectively, a weakly compact $\mathrm{JB}^{*}$-triple $E$ ), the norm closed faces of $\mathcal{B}_{A}$ are determined by finite rank partial isometries in $A$ (respectively, by finite rank tripotents in $E$ ). However, for a general $\mathrm{C}^{*}$-algebra $A$ the maximal proper faces of $\mathcal{B}_{A}$ are determined by minimal partial isometries in $A^{* *}$ (see Section 2 for more details). This is a serious obstacle which makes invalid the arguments in [17,25] in the case of $B(H)$.

To avoid the difficulties mentioned in the previous paragraph, our first geometric result shows that a surjective isometry $f$ from the unit sphere of a $\mathrm{C}^{*}$-algebra $A$ onto the unit sphere of $B(H)$ maps minimal partial isometries in $A$ into minimal partial isometries in $B(H)$ (see Theorem [2.5). Apart from the just commented geometric tools, our arguments are based on techniques of functional analysis and linear algebra. In our main result we prove that, given two complex Hilbert spaces $H$ and $K$, every surjective isometry $f: S(B(K)) \rightarrow S(B(H))$ admits an extension to a surjective complex linear or conjugate linear isometry $T: B(K) \rightarrow B(H)$ (see Theorem 3.2). In the final result we show that the same conclusion remains true when $B(H)$ spaces are replaced by $\ell_{\infty}$-sums of $B(H)$ spaces (see Theorem 3.4). The next natural question beyond these conclusions is whether Tingley's problem admits or not a positive answer for Cartan factors and atomic $\mathrm{JBW}^{*}$-triples.

It should be remarked here that the solution to Tingley's problem for surjective isometries between the unit spheres of $K(H)$-spaces in 17, 25 and the solution presented in this note for surjective isometries between the unit spheres of $B(H)$ spaces are completely independent results.

\section{Surjective isometries Between the Unit SPHeRES of TWo $\mathrm{C}^{*}$-ALGEBRAS}

In this section we carry out a study of the geometric properties of those surjective isometries between the unit spheres of two $\mathrm{C}^{*}$-algebras with special interest on $\mathrm{C}^{*}$ algebras of the form $B(H)$. We begin by gathering some technical results and concepts needed for later purposes.

Proposition 2.1 ([3, Lemma 5.1] and [32, Lemma 3.5]). Let $X, Y$ be Banach spaces, and let $T: S(X) \rightarrow S(Y)$ be a surjective isometry. Then $C$ is a maximal convex subset of $S(X)$ if and only if $T(C)$ is that of $S(Y)$. Then $C$ is a maximal proper (norm-closed) face of $\mathcal{B}_{X}$ if and only if $f(C)$ is a maximal proper (normclosed) face of $\mathcal{B}_{Y}$.

An interesting generalization of the Mazur-Ulam theorem was established by P. Mankiewicz in 22, who proved that, given two convex bodies $V \subset X$ and 
$W \subset Y$, every surjective isometry $g$ from $V$ onto $W$ can be uniquely extended to an affine isometry from $X$ onto $Y$. Consequently, every surjective isometry between the closed unit balls of two Banach spaces $X$ and $Y$ extends uniquely to a real linear isometric isomorphism from $X$ into $Y$.

Let $a$ and $b$ be two elements in a $\mathrm{C}^{*}$-algebra $A$. We recall that $a$ and $b$ are orthogonal ( $a \perp b$ for short) if $a b^{*}=b^{*} a=0$. Symmetric elements in $A$ are orthogonal if and only if their product is zero.

For each element $a$ in a $C^{*}$-algebra $A$, the symbol $|a|$ will denote the element $\left(a^{*} a\right)^{\frac{1}{2}} \in A$. Throughout this note, for each $x \in A, \sigma(x)$ will denote the spectrum of the element $x$. We observe that $\sigma(|a|) \cup\{0\}=\sigma\left(\left|a^{*}\right|\right) \cup\{0\}$, for every $a \in A$. Let $a=v|a|$ be the polar decomposition of $a$ in $A^{* *}$, where $v$ is a partial isometry in $A^{* *}$, which, in general, does not belong to $A$ (compare [27]). It is further known that $v^{*} v$ is the range projection of $|a|(r(|a|)$ for short), and for each $h \in C(\sigma(|a|))$, with $h(0)=0$ the element $v h(|a|) \in A$ (see [1, Lemma 2.1]).

Proposition 2.1 points out the importance of an appropriate description of the maximal proper faces of the closed unit ball $\mathcal{B}_{A}$ of a $\mathrm{C}^{*}$-algebra $A$. A complete study was established by C. A. Akemann and G. K. Pedersen in [2]. When $A$ is a von Neumann algebra, weak*-closed faces in $\mathcal{B}_{A}$ were originally determined by C. M. Edwards and G. T. Rüttimann in [14, who proved that general weak*-closed faces in $\mathcal{B}_{A}$ have the form

$$
F_{v}=v+\left(1-v v^{*}\right) \mathcal{B}_{A}\left(1-v^{*} v\right)=\left\{x \in \mathcal{B}_{A}: x v^{*}=v v^{*}\right\}
$$

for some partial isometry $v$ in $A$. Actually, the mapping $v \mapsto F_{v}$ is an anti-order isomorphism from the complete lattice of partial isometries in $A$ onto the complete lattice of weak*-closed faces of $\mathcal{B}_{A}$, where the partial order in the set of partial isometries of $A$ is given by $v \leq u$ if and only if $u=v+\left(1-v v^{*}\right) u\left(1-v^{*} v\right.$ ) (see [14, Theorem 4.6]).

However, partial isometries in a general $\mathrm{C}^{*}$-algebra $A$ are not enough to determine all the norm-closed faces in $\mathcal{B}_{A}$, even more after recalling the existence of $\mathrm{C}^{*}$-algebras containing no partial isometries. In the general case, certain partial isometries in the second dual, $A^{* *}$, are required to determine the facial structure of $\mathcal{B}_{A}$. We recall that a projection $p$ in $A^{* *}$ is called open if $A \cap\left(p A^{* *} p\right)$ is weak ${ }^{*}$-dense in $p A^{* *} p$ (see [24, §3.11] and [28, §III.6]). A projection $p \in A^{* *}$ is said to be closed if $1-p$ is open. A closed projection $p \in A^{* *}$ is compact if $p \leq x$ for some positive norm-one element $x \in A$. A partial isometry $v \in A^{* *}$ belongs locally to $A$ if $v^{*} v$ is a compact projection and there exists a norm-one element $x$ in $A$ satisfying $v=x v^{*} v$ (compare [2, Remark 4.7]).

It is shown in [2, Lemma 4.8 and Remark 4.11] that "the partial isometries that belong locally to $A$ are obtained by taking an element $x$ in $A$ with norm 1 and polar decomposition $x=u|x|$ (in $A^{* *}$ ), and then letting $v=u e$ for some compact projection $e$ contained in the spectral projection $\chi_{\{1\}}(|x|)$ of $|x|$ corresponding to the eigenvalue $1 "$.

It should be noted that a partial isometry $v$ in $A^{* *}$ belongs locally to $A$ if and only if it is compact in the sense introduced by C. M. Edwards and G. T. Rüttimann in [15, Theorem 5.1].

The facial structure of the unit ball of a $\mathrm{C}^{*}$-algebra is completely described by the following result due to C. A. Akemann and G. K. Pedersen. 
Theorem 2.2 ([2, Theorem 4.10]). Let $A$ be a $C^{*}$-algebra. The norm-closed faces of the unit ball of $A$ have the form

$$
F_{v}=\left(v+\left(1-v v^{*}\right) \mathcal{B}_{A^{* *}}\left(1-v^{*} v\right)\right) \cap \mathcal{B}_{A}=\left\{x \in \mathcal{B}_{A}: x v^{*}=v v^{*}\right\},
$$

for some partial isometry $v$ in $A^{* *}$ belonging locally to A. Actually, the mapping $v \mapsto$ $F_{v}$ is an anti-order isomorphism from the complete lattice of partial isometries in $A^{* *}$ belonging locally to $A$ onto the complete lattice of norm-closed faces of $\mathcal{B}_{A}$.

A non-zero partial isometry $e$ in a $\mathrm{C}^{*}$-algebra $A$ is called minimal if $e e^{*}$ (equivalently, $\left.e^{*} e\right)$ is a minimal projection in $A$, that is, $e e^{*} A e e^{*}=\mathbb{C} e e^{*}$. By Kadison's transitivity theorem minimal partial isometries in $A^{* *}$ belong locally to $A$, and hence every maximal proper face of the unit ball of a $\mathrm{C}^{*}$-algebra $A$ is of the form

$$
\left(v+\left(1-v v^{*}\right) \mathcal{B}_{A^{* *}}\left(1-v^{*} v\right)\right) \cap \mathcal{B}_{A}
$$

for a unique minimal partial isometry $v$ in $A^{* *}$ (compare [2, Remark 5.4 and Corollary 5.5]).

Our main goal in this section is to show that a surjective isometry $f: S(A) \rightarrow$ $S(B)$ between the unit spheres of two $\mathrm{C}^{*}$-algebras maps minimal partial isometries into minimal partial isometries. In a first step we shall show that, for each minimal partial isometry $e$ in $A, 1$ is isolated in the spectrum of $|f(e)|$.

Theorem 2.3. Let $A$ and $B$ be $C^{*}$-algebras, and suppose that $f: S(A) \rightarrow S(B)$ is a surjective isometry. Let $e$ be a minimal partial isometry in $A$. Then 1 is isolated in the spectrum of $|f(e)|$.

Proof. Since $e$ also is a minimal partial isometry in $A^{* *}$ and belongs (locally) to $A$, the set $F_{e}=e+\left(1-e e^{*}\right) \mathcal{B}_{A}\left(1-e^{*} e\right)$ is a maximal proper face of $\mathcal{B}_{A}$. Applying Proposition 2.1 and Theorem 2.2 we deduce the existence of a minimal partial isometry $w$ in $B^{* *}$ such that

(1) $f\left(F_{e}\right)=F_{w}=\left(w+\left(1-w w^{*}\right) \mathcal{B}_{B^{* *}}\left(1-w^{*} w\right)\right) \cap \mathcal{B}_{B}=\left\{x \in \mathcal{B}_{A}: x w^{*}=w w^{*}\right\}$.

Since $f(e) \in f\left(F_{e}\right)=F_{w}$ we have $f(e)=w+\left(1-w w^{*}\right) f(e)\left(1-w^{*} w\right)$.

Arguing by contradiction, we assume that 1 is not isolated in $\sigma(|f(e)|)$. Let $f(e)=r|f(e)|$ denote the polar decomposition of $f(e)$.

By assumptions we can find $t_{0} \in \sigma(|f(e)|)$ satisfying $\frac{3}{\sqrt{10}}<t_{0}<1$. Let us consider the functions $h_{1}$ and $h_{2}$ in the unit sphere of $C_{0}(\sigma(|f(e)|))$ given by

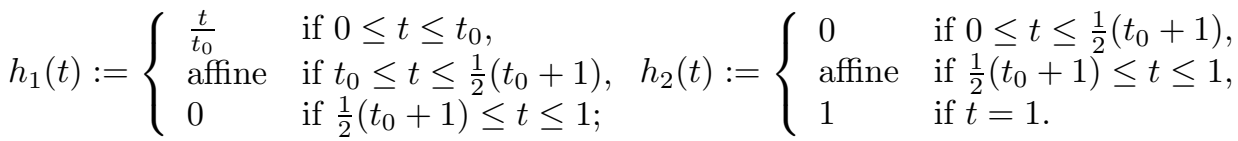

We set $\widehat{x}=r h_{1}(|f(e)|)$ and $\widehat{y}=r h_{2}(|f(e)|)$. Obviously, $h_{1}(|f(e)|)$ and $h_{2}(|f(e)|)$ are positive elements in $S(B)$ satisfying $h_{1}(|f(e)|) h_{2}(|f(e)|)=0$. Since

$$
\widehat{x} \widehat{y}^{*}=r h_{1}(|f(e)|) h_{2}(|f(e)|) r^{*}=0,
$$

and

$$
\widehat{y}^{*} \widehat{x}=h_{2}(|f(e)|) r^{*} r h_{1}(|f(e)|)=h_{2}(|f(e)|) h_{1}(|f(e)|)=0,
$$

it follows that $\hat{x} \perp \hat{y}$.

Let $x=f^{-1}(-\hat{x}) \in S(A)$ and $y=f^{-1}(\hat{y}) \in S(A)$. Since $f$ is an isometry we deduce that

$$
1=\|\hat{x}+\hat{y}\|=\|\hat{y}-(-\hat{x})\|=\|f(y)-f(x)\|=\|y-x\|,
$$


and

$$
1+t_{0}=\|f(e)+\hat{x}\|=\|f(e)-f(x)\|=\|e-x\| .
$$

We recall that, from (1), $f(e)=w+k$ where $k=\left(1-w w^{*}\right) f(e)\left(1-w^{*} w\right)$ satisfies $k^{*} w=0=w k^{*}$, which proves that $k \perp w$. Let $r_{0}$ denote the (unique) partial isometry appearing in the polar decomposition of $k$. Since $r$ is the partial isometry in the polar decomposition of $f(e), w \perp k$, and $f(e)=w+k$, it follows that $r=w+r_{0}$ with $r_{0} \perp w$. We also know that $|f(e)|=w^{*} w+|k|$, and hence a simple application of the continuous functional calculus (having in mind that $h_{2}(1)=1$ ) shows that $h_{2}(|f(e)|)=w^{*} w+h_{2}(|k|)$, with $w^{*} w \perp h_{2}(|k|)$. We therefore have

(2) $\hat{y} w^{*}=r h_{2}(|f(e)|) w^{*}=r w^{*} w w^{*}+r h_{2}(|k|) w^{*}=r w^{*}=\left(w+r_{0}\right) w^{*}=w w^{*}$,

which implies that $\hat{y} \in F_{w}$, and consequently $y=f^{-1}(\hat{y}) \in F_{e}$ (see (1)).

We claim that

$$
\left\|e e^{*}(e-x) e^{*} e\right\|>1 .
$$

The element $e-x$ has norm $1+t_{0}>1$. Suppose that $\left\{H_{i}\right\}_{I}$ is a family of complex Hilbert spaces and $\pi: A \rightarrow \bigoplus_{i}^{\ell_{\infty}} B\left(H_{i}\right)$ is an isometric *-homomorphism with weak*-dense range (we can consider, for example, the atomic representation of $A$ [24, 4.3.7], where the family $I$ is precisely the set of all pure states of $A$ and $\pi$ is the direct sum of all the irreducible representations associated with the pure states [24, Theorem 3.13.2]). For each $j \in I$, let $P_{j}$ denote the projection of $\bigoplus_{i}^{\ell_{\infty}} B\left(H_{i}\right)$ onto $B\left(H_{j}\right)$ and let $\pi_{j}=P_{j} \circ \pi$. Clearly, $\pi_{j}$ is a ${ }^{*}$-homomorphism with weak*-dense range. Since $e$ is a minimal partial isometry, there exists a unique $i_{0} \in I$ such that $\pi_{i_{0}}(e)$ is a non-zero (minimal) partial isometry and $\pi_{j}(e)=0$, for every $j \neq i_{0}$. We also know that $\|x\|=1$, and thus $\left\|\pi_{i_{0}}(e-x)\right\|=1+t_{0}$.

Let $\pi_{i_{0}}(e-x)=u\left|\pi_{i_{0}}(e-x)\right|$ be the polar decomposition of $\pi_{i_{0}}(e-x)$ in $B\left(H_{i_{0}}\right)$. Take $0<\varepsilon<t_{0}-\frac{3}{\sqrt{10}}$. Since $\left\|\left|\pi_{i_{0}}(e-x)\right|\right\|=1+t_{0}$, we can find a minimal projection $q=\xi \otimes \xi \in B\left(H_{i_{0}}\right)$ with $\|\xi\|=1$ in $H_{i_{0}}$ satisfying $q \leq u^{*} u$ and

$$
1+t_{0}-\varepsilon<\left\langle\left|\pi_{i_{0}}(e-x)\right|(\xi) / \xi\right\rangle,
$$

and

$$
\begin{aligned}
1+t_{0}- & \varepsilon\left\langle\left\langle\left|\pi_{i_{0}}(e-x)\right|(\xi) / \xi\right\rangle \leq\left\langle\left|\pi_{i_{0}}(e-x)\right|(\xi) /\left|\pi_{i_{0}}(e-x)\right|(\xi)\right\rangle^{\frac{1}{2}}\|\xi\|\right. \\
= & \left\langle\left|\pi_{i_{0}}(e-x)\right|^{2}(\xi) / \xi\right\rangle^{\frac{1}{2}}=\left\langle\pi_{i_{0}}(e-x)^{*} \pi_{i_{0}}(e-x)(\xi) / \xi\right\rangle^{\frac{1}{2}} .
\end{aligned}
$$

The element $v=u q$ is a minimal partial isometry in $B\left(H_{i_{0}}\right)$.

We observe that $\pi(e)=\pi_{i_{0}}(e)$ and $v$ are not orthogonal. Otherwise, $\pi_{i_{0}}(e)^{*} \pi_{i_{0}}(e)$ $\perp v^{*} v=q$, and hence $\pi_{i_{0}}(e) q=0=q \pi_{i_{0}}(e)^{*}$, which, by (5), implies that

$$
\begin{gathered}
\left(1+t_{0}-\varepsilon\right)^{2}<\left\langle\pi_{i_{0}}(e-x)^{*} \pi_{i_{0}}(e-x)(\xi) / \xi\right\rangle=\left\langle q \pi_{i_{0}}(e-x)^{*} \pi_{i_{0}}(e-x) q(\xi) / \xi\right\rangle \\
=\left\langle q \pi_{i_{0}}(x)^{*} \pi_{i_{0}}(x) q(\xi) / \xi\right\rangle \leq\left\|\pi_{i_{0}}(x)^{*} \pi_{i_{0}}(x)\right\|=\left\|\pi_{i_{0}}(x)\right\|^{2} \leq\|x\|^{2}=1,
\end{gathered}
$$

which is impossible.

Therefore, $\pi_{i_{0}}(e)$ and $v$ are two minimal partial isometries in $B\left(H_{i_{0}}\right)$ which are not orthogonal. They must be of the form $\pi_{i_{0}}(e)=\eta_{1} \otimes \xi_{1}$ and $v=\widetilde{\eta}_{1} \otimes \widetilde{\xi}_{1}$ for suitable $\xi_{1}, \eta_{1}, \widetilde{\xi}_{1}, \widetilde{\eta}_{1} \in S\left(H_{i_{0}}\right)$ with $\left|\left\langle\xi_{1} / \widetilde{\xi}_{1}\right\rangle\right|+\left|\left\langle\widetilde{\eta}_{1} / \eta_{1}\right\rangle\right| \neq 0$. Let us consider two orthonormal systems $\left\{\eta_{1}, \eta_{2}\right\}$ and $\left\{\xi_{1}, \xi_{2}\right\}$ such that

$$
v=\alpha \pi_{i_{0}}(e)+\beta v_{12}+\delta v_{22}+\gamma v_{21},
$$

where $\pi_{i_{0}}(e):=v_{11}=\eta_{1} \otimes \xi_{1}, v_{12}=\eta_{2} \otimes \xi_{1}, v_{21}=\eta_{1} \otimes \xi_{2}, v_{22}=\eta_{2} \otimes \xi_{2}, \alpha=$ $\left\langle\xi_{1} / \widetilde{\xi}_{1}\right\rangle\left\langle\widetilde{\eta}_{1} / \eta_{1}\right\rangle, \beta=\left\langle\xi_{1} / \widetilde{\xi}_{1}\right\rangle\left\langle\widetilde{\eta}_{1} / \eta_{2}\right\rangle, \gamma=\left\langle\xi_{2} / \widetilde{\xi}_{1}\right\rangle\left\langle\widetilde{\eta}_{1} / \eta_{1}\right\rangle, \delta=\left\langle\xi_{2} / \widetilde{\xi}_{1}\right\rangle\left\langle\widetilde{\eta}_{1} / \eta_{2}\right\rangle \in \mathbb{C}$. It 
is easy to check that $|\alpha|^{2}+|\beta|^{2}+|\gamma|^{2}+|\delta|^{2}=\left|\left\langle\xi_{1} / \widetilde{\xi}_{1}\right\rangle\right|^{2}\left\|\widetilde{\eta}_{1}\right\|^{2}+\left|\left\langle\xi_{2} / \widetilde{\xi}_{1}\right\rangle\right|^{2}\left\|\widetilde{\eta}_{1}\right\|^{2}=$ $\left\|\widetilde{\xi}_{1}\right\|^{2}=1$, and $\alpha \delta=\beta \gamma$.

For each $(i, j) \in\{1,2\}^{2}$, let $\varphi_{i j} \in S\left(B\left(H_{i_{0}}\right)_{*}\right)$ be the unique extreme point of the unit ball $\mathcal{B}_{B\left(H_{i_{0}}\right)_{*}}$ defined by $\varphi_{i j}(z):=\left\langle z\left(\xi_{i}\right) / \eta_{j}\right\rangle\left(z \in B\left(H_{i_{0}}\right)\right)$. We shall also consider $\varphi_{v} \in S\left(B\left(H_{i_{0}}\right)_{*}\right)$, defined by $\varphi_{v}(z):=\left\langle z\left(\widetilde{\xi}_{1}\right) / \widetilde{\eta}_{1}\right\rangle\left(z \in B\left(H_{i_{0}}\right)\right)$. Each $\varphi_{i j}$ is supported by $v_{i j} \in S\left(B\left(H_{i_{0}}\right)\right)$, while $\varphi_{v}$ is supported by $v$.

Clearly, the identity

$$
\varphi_{v}\left(\pi_{i_{0}}(e)\right)=\left\langle\pi_{i_{0}}(e)\left(\widetilde{\xi}_{1}\right) / \widetilde{\eta}_{1}\right\rangle=\left\langle\widetilde{\xi}_{1} / \xi_{1}\right\rangle\left\langle\eta_{1} / \widetilde{\eta}_{1}\right\rangle=\bar{\alpha}
$$

holds, and similarly we have

$$
\varphi_{v} \pi_{i_{0}}(x)=\bar{\alpha} z_{11}+\bar{\beta} z_{12}+\bar{\delta} z_{22}+\bar{\gamma} z_{21},
$$

where $z_{i j}=\varphi_{i j} \pi_{i_{0}}(x)$ for all $(i, j) \in\{1,2\}^{2}$. We also know that $\widetilde{\xi}_{1} \otimes \widetilde{\xi}_{1}=v^{*} v=$ $q=\xi \otimes \xi \leq u^{*} u$, and thus $\widetilde{\xi}_{1}=\mu_{0} \xi$, for a suitable $\mu_{0} \in \mathbb{C}$ with $\left|\mu_{0}\right|=1$. We deduce from (41) that

$$
\begin{gathered}
1+t_{0}-\varepsilon<\left\langle\left|\pi_{i_{0}}(e-x)\right|(\xi) / \xi\right\rangle=\left\langle u^{*} u\left|\pi_{i_{0}}(e-x)\right|(\xi) / \xi\right\rangle=\left\langle u\left|\pi_{i_{0}}(e-x)\right|(\xi) / u q(\xi)\right\rangle \\
=\left\langle\pi_{i_{0}}(e-x)(\xi) / v(\xi)\right\rangle=\left\langle\pi_{i_{0}}(e-x)\left(\widetilde{\xi}_{1}\right) / v\left(\widetilde{\xi}_{1}\right)\right\rangle=\left\langle\pi_{i_{0}}(e-x)\left(\widetilde{\xi}_{1}\right) / \widetilde{\eta}_{1}\right\rangle=\varphi_{v}\left(\pi_{i_{0}}(e-x)\right) \\
=\varphi_{v}\left(\pi_{i_{0}}(e)\right)+\varphi_{v}\left(\pi_{i_{0}}(x)\right) \leq|\alpha|+\left|\varphi_{v}\left(\pi_{i_{0}}(x)\right)\right| \leq|\alpha|+1,
\end{gathered}
$$

which proves

$$
t_{0}-\varepsilon<|\alpha| \leq 1 \text {. }
$$

Now, the equality $|\alpha|^{2}+|\beta|^{2}+|\gamma|^{2}+|\delta|^{2}=1$ implies that

$$
|\beta|^{2},|\gamma|^{2},|\delta|^{2} \leq 1-\left(t_{0}-\varepsilon\right)^{2}<\frac{1}{10},
$$

and since $\left|z_{i j}\right| \leq 1$, we have

$$
\begin{aligned}
& 1+t_{0}-\varepsilon<\varphi_{v} \pi_{i_{0}}(e-x)=\left|\varphi_{v} \pi_{i_{0}}(e-x)\right|=\left|\bar{\alpha}-\left(\bar{\alpha} z_{11}+\bar{\beta} z_{12}+\bar{\delta} z_{22}+\bar{\gamma} z_{21}\right)\right| \\
\leq & |\alpha|\left|1-z_{11}\right|+\left|z_{12}\right||\beta|+\left|z_{21}\right||\gamma|+\left|z_{22}\right||\delta| \leq\left|1-z_{11}\right|+|\beta|+|\gamma|+|\delta| \leq\left|1-z_{11}\right|+\frac{3}{\sqrt{10}} .
\end{aligned}
$$

Let us observe that $\pi_{i_{0}}(e) \pi_{i_{0}}(e)^{*}=v_{11} v_{11}^{*}=\eta_{1} \otimes \eta_{1}$ and $\pi_{i_{0}}(e)^{*} \pi_{i_{0}}(e)=v_{11}^{*} v_{11}=$ $\xi_{1} \otimes \xi_{1}$. Therefore

$$
\begin{gathered}
1<1+t_{0}-\varepsilon-\frac{3}{\sqrt{10}} \leq\left|1-z_{11}\right|=\left|\varphi_{11} \pi_{i_{0}}(e-x)\right| \\
=\left|\varphi_{11}\left(\left(\pi_{i_{0}}(e) \pi_{i_{0}}(e)^{*}\right) \pi_{i_{0}}(e-x)\left(\pi_{i_{0}}(e)^{*} \pi_{i_{0}}(e)\right)\right)\right| \\
\leq\left\|\left(\pi_{i_{0}}(e) \pi_{i_{0}}(e)^{*}\right) \pi_{i_{0}}(e-x)\left(\pi_{i_{0}}(e)^{*} \pi_{i_{0}}(e)\right)\right\| \\
=\left\|\pi_{i_{0}}\left(\left(e e^{*}\right)(e-x)\left(e^{*} e\right)\right)\right\| \leq\left\|\left(e e^{*}\right)(e-x)\left(e^{*} e\right)\right\|,
\end{gathered}
$$

which proves the claim in (3).

Finally, since $y \in F_{e}=e+\left(1-e e^{*}\right) \mathcal{B}_{A}\left(1-e^{*} e\right)$ we can write

$$
y=e+\left(1-e e^{*}\right) y\left(1-e^{*} e\right),
$$

and we deduce from (3) that

$$
\begin{gathered}
1=\|y-x\| \geq\left\|e e^{*}(y-x) e^{*} e\right\|=\left\|e e^{*}\left(e+\left(1-e e^{*}\right) y\left(1-e^{*} e\right)-x\right) e^{*} e\right\| \\
=\left\|e e^{*}(e-x) e^{*} e\right\|>1,
\end{gathered}
$$

leading to the desired contradiction. 
In the problem of dealing with maximal faces of the unit ball of a $\mathrm{C}^{*}$-algebra $A$ we need to handle minimal partial isometries in $A^{* *}$ (compare Theorem 2.2). We now present a technical result, which will be used later to facilitate the arguments depending on the facial structure of $\mathcal{B}_{A}$.

Lemma 2.4. Let $A$ be a $C^{*}$-algebra. The following statements hold:

(a) Every minimal projection $p$ in $A^{* *} \backslash A$ is orthogonal to all minimal projections in $A$.

(b) Every minimal partial isometry $u$ in $A^{* *} \backslash A$ is orthogonal to all minimal partial isometries in $A$.

Proof. (a) Suppose $p$ is a minimal projection in $A^{* *} \backslash A$. Let $q$ denote a minimal projection in $A$. Arguing by contradiction we assume that $p q \neq 0$.

As in the proof of Theorem 2.3 let $\pi: A \rightarrow \bigoplus_{i}^{\ell_{\infty}} B\left(H_{i}\right)$ be an isometric *homomorphism with weak*-dense range, where $\left\{H_{i}\right\}_{I}$ is a family of complex Hilbert spaces (consider, for example, the atomic representation of $A$ [24, 4.3.7]). By the weak*-density of $A$ in $\bigoplus_{i}^{\ell_{\infty}} B\left(H_{i}\right)$ and the separate weak*-continuity of the product of every von Neumann algebra, $\pi(q)$ is a minimal projection in $\bigoplus_{i}^{\ell_{\infty}} B\left(H_{i}\right)$. Clearly, the images of the mappings $L_{\pi(q)}: x \mapsto \pi(q) x$ and $R_{\pi(q)}: x \mapsto x \pi(q)$ $\left(\forall x \in \bigoplus_{i}^{\ell_{\infty}} B\left(H_{i}\right)\right)$ are contained in suitable Hilbert spaces. It follows that the left and right multiplication operators $L_{q}$ and $R_{q}$ by $q$ on $A$ factors through a Hilbert space, and thus they are weakly compact (compare [6]). Consequently, the spaces $(1-q) A q, q A(1-q)$, and $q A q=\mathbb{C} q$ are all reflexive. Applying the Krein-Šmulian theorem we deduce that $(1-q) A q, q A(1-q)$, and $q A q=\mathbb{C} q$ are weak*-closed in $A^{* *}$, showing that

$$
(1-q) A^{* *} q=(1-q) A q, q A^{* *}(1-q)=q A(1-q) \subseteq A \text {, and } q A^{* *} q=q A q=\mathbb{C} q .
$$

We now recall a useful matricial representation theorem. Let $C$ denote the $\mathrm{C}^{*}$ subalgebra of $A^{* *}$ generated by $p$ and $q$. Since $p$ and $q$ are minimal projections in $A^{* *}$, Theorem 1.3 in [26] (see also [23, §3]) assures the existence of $t \in$ $[0,1]$ and a ${ }^{*}$-isomorphism $\Phi: C \rightarrow M_{2}(\mathbb{C})$ such that $\Phi(q)=\left(\begin{array}{ll}1 & 0 \\ 0 & 0\end{array}\right)$ and $\Phi(p)=\left(\begin{array}{cc}t & \sqrt{t(1-t)} \\ \sqrt{t(1-t)} & 1-t\end{array}\right)$. Since $p q \neq 0$ we know that $t \neq 0$. Clearly, $\Phi^{-1}\left(\begin{array}{ll}0 & 1 \\ 1 & 0\end{array}\right) \in q C(1-q) \oplus(1-q) C q \subset A$, and $\Phi^{-1}\left(\begin{array}{ll}1 & 0 \\ 0 & 0\end{array}\right)=q \in A$. Then $\Phi^{-1}\left(\begin{array}{ll}0 & 0 \\ 0 & 1\end{array}\right)=\Phi^{-1}\left(\left(\begin{array}{ll}0 & 1 \\ 1 & 0\end{array}\right)\left(\begin{array}{ll}1 & 0 \\ 0 & 0\end{array}\right)\left(\begin{array}{ll}0 & 1 \\ 1 & 0\end{array}\right)\right) \in \Phi^{-1}(\Phi(A \cap C))=A \cap C$. By linearity $p=\Phi^{-1}\left(\begin{array}{cc}t & \sqrt{t(1-t)} \\ \sqrt{t(1-t)} & 1-t\end{array}\right) \in A$ which is impossible.

(b) Suppose now that $u$ is a minimal partial isometry in $A^{* *} \backslash A$ and $v$ is a minimal partial isometry in $A$. We shall first show that $u u^{*}, u^{*} u \in A^{* *} \backslash A$. Indeed, since every minimal partial isometry in $A^{* *}$ belongs locally to $A$ (compare Kadison's transitivity theorem and [2, Remark 5.4 and Corollary 5.5]), there exists a norm 1 element $x \in A$ satisfying $x=u+\left(1-u u^{*}\right) x\left(1-u^{*} u\right)$. If $u u^{*}$ (respectively, $u^{*} u$ ) lies in $A$, then $u=u u^{*} x \in A$ (respectively, $u=x u^{*} u \in A$ ) which is impossible.

We have therefore shown that $u u^{*}, u^{*} u \in A^{* *} \backslash A$ are minimal projections, while $v v^{*}, v^{*} v$ are minimal projections in $A$. It follows from $(a)$ that $u u^{*}, u^{*} u \perp v v^{*}, v^{*} v$. 
Finally, the identities $u^{*} v=u^{*} u u^{*} v v^{*} v=0$ and $v u^{*}=v v^{*} v u^{*} u u^{*}=0$ prove that $u \perp v$.

We are now in position to show that a surjective isometry between the unit spheres of two $\mathrm{C}^{*}$-algebras maps minimal partial isometries to minimal partial isometries.

Theorem 2.5. Let $A$ be a $C^{*}$-algebra, and let $H$ be a complex Hilbert space. Suppose that $f: S(A) \rightarrow S(B(H))$ is a surjective isometry. Let e be a minimal partial isometry in $A$. Then $f(e)$ is a minimal partial isometry in $B(H)$. Moreover, there exists a surjective real linear isometry

$$
T_{e}:\left(1-e e^{*}\right) A\left(1-e^{*} e\right) \rightarrow\left(1-f(e) f(e)^{*}\right) B(H)\left(1-f(e)^{*} f(e)\right)
$$

such that

$$
f(e+x)=f(e)+T_{e}(x) \text { for all } x \text { in } \mathcal{B}_{\left(1-e e^{*}\right) A\left(1-e^{*} e\right)} .
$$

In particular the restriction of $f$ to the face $F_{e}=e+\left(1-e e^{*}\right) \mathcal{B}_{A}\left(1-e^{*} e\right)$ is a real affine function.

Proof. Arguing as in the beginning of the proof of Theorem 2.3 , the set

$$
F_{e}=e+\left(1-e e^{*}\right) \mathcal{B}_{A}\left(1-e^{*} e\right)
$$

is a maximal proper face of $\mathcal{B}_{A}$, and thus, by Proposition 2.1 and Theorem 2.2 . there exists a minimal partial isometry $w$ in $B(H)^{* *}$ such that

$$
f\left(F_{e}\right)=F_{w}=\left(w+\left(1-w w^{*}\right) \mathcal{B}_{B(H)^{* *}}\left(1-w^{*} w\right)\right) \cap \mathcal{B}_{B(H)} .
$$

We claim that $w \in B(H)$. Suppose, on the contrary that $w \in B(H)^{* *} \backslash B$.

Theorem 2.3 implies that 1 is an isolated point in $\sigma(|f(e)|)$, and hence the function $\chi_{\{1\}}$ belongs to $C_{0}(\sigma(|f(e)|))$. Let $f(e)=r|f(e)|$ denote the polar decomposition of $f(e)$. An application of the continuous functional calculus proves that $\hat{v}=$ $r \chi_{\{1\}}(|f(e)|)$ is a partial isometry in $B(H)$. Furthermore, since $f(e) \in f\left(F_{e}\right)=F_{w}$, we deduce that $\hat{v} \in F_{w}$ and

$$
\hat{v}=w+\left(1-w w^{*}\right) \hat{v}\left(1-w^{*} w\right)
$$

(compare the arguments in the proof of (2) on page 66).

In $B(H)$ we can always find a minimal partial isometry $\hat{w} \in B(H)$ satisfying

$$
\hat{v}=\hat{w}+\left(1-\hat{w} \hat{w}^{*}\right) \hat{v}\left(1-\hat{w}^{*} \hat{w}\right) .
$$

Since, by assumptions $w \in B(H)^{* *} \backslash B(H)$, Lemma 2.4 implies that $w \perp \hat{w}$,

$$
\hat{w}=\left(1-w w^{*}\right) \hat{w}\left(1-w^{*} w\right)
$$

and hence, by (7) we get

$$
\hat{v}-\hat{w}=w+\left(1-w w^{*}\right)(\hat{v}-\hat{w})\left(1-w^{*} w\right) .
$$

By hypothesis,

$$
2=\|f(e)+\hat{w}\|=\|f(e)-(-\hat{w})\|=\left\|e-f^{-1}(-\hat{w})\right\|,
$$

where $e$ is a minimal partial isometry in $A$. Proposition 2.2 in 17. proves that

$$
\hat{e}=f^{-1}(-\hat{w})=-e+\left(1-e e^{*}\right) \hat{e}\left(1-e^{*} e\right) .
$$

By construction $f(e)=\hat{v}+\left(1-\hat{v} \hat{v}^{*}\right) f(e)\left(1-\hat{v}^{*} \hat{v}\right)$, and by (8),

$$
f(e)=\hat{w}+\left(1-\hat{w} \hat{w}^{*}\right) \hat{v}\left(1-\hat{w}^{*} \hat{w}\right)+\left(1-\hat{v} \hat{v}^{*}\right) f(e)\left(1-\hat{v}^{*} \hat{v}\right),
$$


and consequently $\|f(e)-\hat{w}\| \leq 1$. Having in mind that

$$
f(e)-\hat{w}=f(e)-\left(1-w w^{*}\right) \hat{w}\left(1-w^{*} w\right) \in F_{w}+\left(1-w w^{*}\right) \mathcal{B}_{B(H)}\left(1-w^{*} w\right)
$$

we get

$$
f(e)-\hat{w}=w+\left(1-w w^{*}\right)(f(e)-\hat{w})\left(1-w^{*} w\right) \in F_{w} .
$$

We deduce from (6) that $z=f^{-1}(f(e)-\hat{w}) \in F_{e}$, and thus $z=e+\left(1-e e^{*}\right) z\left(1-e^{*} e\right)$, which leads to

$$
\begin{gathered}
1=\|f(e)\|=\|f(e)-\hat{w}+\hat{w}\|=\|(f(e)-\hat{w})-(-\hat{w})\|=\|f(z)-f(\hat{e})\| \\
=\|z-\hat{e}\|=\left\|e+\left(1-e e^{*}\right) z\left(1-e^{*} e\right)-\left(-e+\left(1-e e^{*}\right) \hat{e}\left(1-e^{*} e\right)\right)\right\| \\
=\left\|2 e+\left(1-e e^{*}\right)(z-\hat{e})\left(1-e^{*} e\right)\right\|=2,
\end{gathered}
$$

and hence to a contradiction. Therefore, $w \in B(H)$, and

$$
F_{w}=w+\left(1-w w^{*}\right) \mathcal{B}_{B(H)}\left(1-w^{*} w\right) .
$$

We can argue now as in the proof of [25, Proposition 3.1] to conclude. We insert a short argument here for completeness reasons. We have established that

$$
f\left(e+\mathcal{B}_{\left(1-e e^{*}\right) A\left(1-e^{*} e\right)}\right)=f\left(F_{e}\right)=F_{w}=w+\mathcal{B}_{\left(1-w w^{*}\right) B(H)\left(1-w^{*} w\right)} .
$$

Let $\mathcal{T}_{x_{0}}$ denote the translation with respect to $x_{0}$, that is, $\mathcal{T}_{x_{0}}(x)=x+x_{0}$. The mapping $f_{e}=\left.\left.\left.\mathcal{T}_{w}^{-1}\right|_{f\left(F_{e}\right)} \circ f\right|_{F_{e}} \circ \mathcal{T}_{e}\right|_{\mathcal{B}_{\left(1-e e^{*}\right) A\left(1-e^{*} e\right)}}$ is a surjective isometry from $\mathcal{B}_{\left(1-e e^{*}\right) A\left(1-e^{*} e\right)}$ onto $\mathcal{B}_{\left(1-w w^{*}\right) B(H)\left(1-w^{*} w\right)}$. Mankiewicz's theorem (see [22]) implies the existence of a surjective real linear isometry $T_{e}:\left(1-e e^{*}\right) A\left(1-e^{*} e\right) \rightarrow$ $\left(1-w w^{*}\right) B(H)\left(1-w^{*} w\right)$ such that $f_{e}=\left.T_{e}\right|_{S\left(\left(1-e e^{*}\right) A\left(1-e^{*} e\right)\right)}$ and hence

$$
f(e+x)=w+T_{e}(x) \text { for all } x \text { in } \mathcal{B}_{\left(1-e e^{*}\right) A\left(1-e^{*} e\right)} .
$$

In particular, $f(e)=w$.

For the final statement we simply write

$$
\left.f\right|_{F_{e}}=\left.\left.\mathcal{T}_{w}\right|_{\mathcal{B}_{\left(1-w w^{*}\right) B(H)\left(1-w^{*} w\right)}} \circ f_{e} \circ \mathcal{T}_{e}^{-1}\right|_{F_{e}}=\left.\left.\mathcal{T}_{w}\right|_{\mathcal{B}_{\left(1-w w^{*}\right) B(H)\left(1-w^{*} w\right)}} \circ T_{e} \circ \mathcal{T}_{e}^{-1}\right|_{F_{e}}
$$

as a composition of real affine functions.

The next technical lemma is obtained with basic techniques of linear algebra.

Lemma 2.6. Let $H$ be a complex Hilbert space, and let $v_{1}, v_{2}, v_{3}, e_{1}$, and $e_{2}$ be minimal partial isometries in $B(H)$ satisfying $e_{1} \perp e_{2}, v_{1} \perp v_{2}, v_{1} \perp v_{3}$,

$$
-v_{1}+v_{3}=e_{1}-e_{2} \quad \text { and } \quad v_{1}+v_{2}=e_{1}+e_{2} .
$$

Then $v_{1}=e_{2}$ and $v_{2}=e_{1}=v_{3}$.

Proof. Since $v_{1} \perp v_{2}, v_{3}$, by multiplying the identities $-v_{1}+v_{3}=e_{1}-e_{2}$ and $v_{1}+v_{2}=e_{1}+e_{2}$ on the left by $v_{1}^{*}$ we get

$$
-v_{1}^{*} v_{1}=v_{1}^{*} e_{1}-v_{1}^{*} e_{2}, \quad \text { and } \quad v_{1}^{*} v_{1}=v_{1}^{*} e_{1}+v_{1}^{*} e_{2},
$$

which shows that $v_{1}^{*} e_{1}=0$. Multiplying by $v_{1}^{*}$ on the right we prove $e_{1} v_{1}^{*}=0$. We have therefore shown that $v_{1} \perp e_{1}$.

Applying that $e_{1} \perp e_{2}$ and $v_{1} \perp v_{2}$ we get $e_{1} e_{1}^{*}+e_{2} e_{2}^{*}=v_{1} v_{1}^{*}+v_{2} v_{2}^{*}$, where $e_{1} e_{1}^{*}$ and $v_{1} v_{1}^{*}$ are orthogonal rank 1 projections. Thus, $e_{1} e_{1}^{*}=e_{1} e_{1}^{*} v_{2} v_{2}^{*}$, and by minimality $v_{2} v_{2}^{*}=e_{1} e_{1}^{*}$. We can similarly prove $v_{2}^{*} v_{2}=e_{1}^{*} e_{1}$. Finally,

$$
v_{2}=v_{2} v_{2}^{*} v_{2}=v_{2} v_{2}^{*}\left(v_{1}+v_{2}\right)=v_{2} v_{2}^{*}\left(e_{1}+e_{2}\right)=e_{1} e_{1}^{*}\left(e_{1}+e_{2}\right)=e_{1},
$$

and the rest is clear. 
Next, we shall establish several consequences of the above theorem.

Theorem 2.7. Let $f: S(B(K)) \rightarrow S(B(H))$ be a surjective isometry where $H$ and $K$ are complex Hilbert spaces with dimension greater than or equal to 3 . Then the following statements hold:

(a) For each minimal partial isometry $v$ in $B(K)$, the mapping

$$
T_{v}:\left(1-v v^{*}\right) B(K)\left(1-v v^{*}\right) \rightarrow\left(1-f(v) f(v)^{*}\right) B(H)\left(1-f(v)^{*} f(v)\right)
$$

given by Theorem 2.5 is complex linear or conjugate linear.

(b) For each minimal partial isometry $v$ in $B(K)$ we have $f(-v)=-f(v)$ and $T_{v}=T_{-v}$. Furthermore, $T_{v}$ is weak ${ }^{*}$-continuous and $f(e)=T_{v}(e)$ for every minimal partial isometry $e \in\left(1-v v^{*}\right) B(K)\left(1-v^{*} v\right)$.

(c) For each minimal partial isometry $v$ in $B(K)$ the equality $f(w)=T_{v}(w)$ holds for every partial isometry $w \in\left(1-v v^{*}\right) B(K)\left(1-v^{*} v\right) \backslash\{0\}$.

(d) Let $w_{1}, \ldots, w_{n}$ be mutually orthogonal non-zero partial isometries in $B(K)$, and let $\lambda_{1}, \ldots, \lambda_{n}$ be positive real numbers with $\lambda_{1}=1$, and $\lambda_{j} \leq 1$ for all j. Then

$$
f\left(\sum_{j=1}^{n} \lambda_{j} w_{j}\right)=\sum_{j=1}^{n} \lambda_{j} f\left(w_{j}\right) .
$$

(e) For each minimal partial isometry $v$ in $B(K)$ we have $f(x)=T_{v}(x)$ for every $x \in S\left(\mathcal{B}_{\left(1-v v^{*}\right) B(K)\left(1-v^{*} v\right)}\right)$.

$(f)$ For each partial isometry $w$ in $B(K)$ the element $f(w)$ is a partial isometry.

(g) Suppose $v_{1}, v_{2}$ are mutually orthogonal minimal partial isometries in $B(K)$; then $T_{v_{1}}(x)=T_{v_{2}}(x)$ for every $x \in\left(1-v_{1} v_{1}^{*}\right) B(K)\left(1-v_{1} v_{1}^{*}\right) \cap(1-$ $\left.v_{2} v_{2}^{*}\right) B(K)\left(1-v_{2} v_{2}^{*}\right)$.

(h) Suppose $v_{1}, v_{2}$ are mutually orthogonal minimal partial isometries in $B(K)$; then exactly one of the following statements holds:

(1) The mappings $T_{v_{1}}$ and $T_{v_{2}}$ are complex linear.

(2) The mappings $T_{v_{1}}$ and $T_{v_{2}}$ are conjugate linear.

Proof. (a) Let $v$ be a minimal partial isometry in $B(K)$. Suppose that

$$
T_{v}:\left(1-v v^{*}\right) B(K)\left(1-v^{*} v\right) \rightarrow\left(1-f(v) f(v)^{*}\right) B(H)\left(1-f(v)^{*} f(v)\right)
$$

is the surjective real linear isometry given by Theorem 2.5. Having in mind that (1$\left.v v^{*}\right) B(K)\left(1-v^{*} v\right) \cong B\left(\left(1-v^{*} v\right)(K),\left(1-v v^{*}\right)(K)\right)$ and $\left(1-f(v) f(v)^{*}\right) B(H)(1-$ $\left.f(v)^{*} f(v)\right) \cong B\left(\left(1-f(v)^{*} f(v)\right)(H),\left(1-f(v) f(v)^{*}\right)(H)\right)$ are Cartan factors of type 1 and rank $\geq 2$, Proposition 2.6 in [5] assures that $T_{v}$ is complex linear or conjugate linear.

(b) We keep the notation in $(a)$. By Theorem 2.5 $f(v)$ and $f(-v)$ are minimal partial isometries. By assumptions $\|f(v)-f(-v)\|=\|v+v\|=2$, and hence by [25. Lemmas 3.4 and 3.5] or [17, Proposition 2.2] we have $f(-v)=-f(v)$. Let $T_{v}, T_{-v}:\left(1-v v^{*}\right) B(K)\left(1-v^{*} v\right) \rightarrow\left(1-f(v) f(v)^{*}\right) B(H)\left(1-f(v)^{*} f(v)\right)$ be the surjective real linear isometries given by Theorem [2.5. Lemma 2.5 in [5] proves that $T_{v}$ and $T_{-v}$ both are weak*-continuous, while [5, Proposition 2.6] implies that $T_{v}$ and $T_{-v}$ preserve products of the form $(a, b, c) \mapsto a b^{*} c+c b^{*} a(a, b, c \in$ $\left.\left(1-v v^{*}\right) B(K)\left(1-v^{*} v\right)\right)$.

By the hypothesis on $H$, we can find another minimal partial isometry $e \in$ $\left(1-v v^{*}\right) B(K)\left(1-v^{*} v\right)$. Since $e+v, e-v \in F_{e}$, applying Theorem 2.5 we deduce 
that

$$
f(v)+T_{v}(e)=f(e+v)=f(e)+T_{e}(v)
$$

and

$$
-f(v)+T_{-v}(e)=f(-v)+T_{-v}(e)=f(e-v)=f(e)-T_{e}(v),
$$

where $T_{v}(e), T_{-v}(e)$ and $T_{e}(v)$ are minimal partial isometries with $f(v) \perp T_{v}(e)$, $-f(v)=f(-v) \perp T_{-v}(e)$, and $f(e) \perp T_{e}(v)$. It follows from Lemma 2.6 above that $T_{e}(v)=f(v), T_{v}(e)=f(e)=T_{-v}(e)$, and $f(-v)=-T_{e}(v)=-f(v)$.

We have also shown that $T_{v}(e)=T_{-v}(e)$ for every minimal partial isometry $e \in\left(1-v v^{*}\right) B(K)\left(1-v^{*} v\right)$. That is, $T_{v}$ and $T_{-v}$ are surjective complex linear or conjugate linear surjective isometries between Cartan factors of type 1 and rank $\geq 2$. Since $T_{v}$ and $T_{-v}$ coincide on minimal partial isometries, we deduce by linearity that $T_{v}$ and $T_{-v}$ both are complex linear or conjugate linear and coincide on finite linear combinations of mutually orthogonal minimal partial isometries. Finally, since $\left(1-v v^{*}\right) B(K)\left(1-v^{*} v\right) \cong B\left(\left(1-v^{*} v\right)(K),\left(1-v v^{*}\right)(K)\right)$ coincides with the weak*-closed span of its minimal tripotents, we conclude that $T_{v}=T_{-v}$.

(c) Let $w$ be a non-zero partial isometry in $\left(1-v v^{*}\right) B(K)\left(1-v^{*} v\right)$. Take a minimal partial isometry $w_{0}$ such that $w=w_{0}+\left(1-w_{0} w_{0}^{*}\right) w\left(1-w_{0}^{*} w_{0}\right)$. We set $w_{0}^{\perp}=\left(1-w_{0} w_{0}^{*}\right) w\left(1-w_{0}^{*} w_{0}\right)$. Applying Theorem 2.5] and $(b)$ we get

$$
\begin{gathered}
f(v)+T_{v}(w)=f(v+w)=f\left(v+w_{0}+w_{0}^{\perp}\right)=f\left(w_{0}\right)+T_{w_{0}}(v)+T_{w_{0}}\left(w_{0}^{\perp}\right) \\
=f\left(w_{0}\right)+f(v)+T_{w_{0}}\left(w_{0}^{\perp}\right)=f(v)+f\left(w_{0}+w_{0}^{\perp}\right)=f(v)+f(w),
\end{gathered}
$$

which proves $(c)$.

(d) Let $w_{1}, \ldots, w_{n}$ be mutually orthogonal non-zero partial isometries in $B(K)$, and let $\lambda_{1}, \ldots, \lambda_{n}$ be positive real numbers with $\lambda_{1}=1$. Pick again a minimal partial isometry $w_{0}$ such that $w_{1}=w_{0}+\left(1-w_{0} w_{0}^{*}\right) w_{1}\left(1-w_{0}^{*} w_{0}\right)$. Theorem 2.5 proves that

$$
\begin{gathered}
f\left(\sum_{j=1}^{n} \lambda_{j} w_{j}\right)=f\left(w_{0}+\left(1-w_{0} w_{0}^{*}\right) w_{1}\left(1-w_{0}^{*} w_{0}\right)+\sum_{j=2}^{n} \lambda_{j} w_{j}\right) \\
=f\left(w_{0}\right)+T_{w_{0}}\left(\left(1-w_{0} w_{0}^{*}\right) w_{1}\left(1-w_{0}^{*} w_{0}\right)+\sum_{j=2}^{n} \lambda_{j} w_{j}\right) \\
=f\left(w_{0}\right)+T_{w_{0}}\left(\left(1-w_{0} w_{0}^{*}\right) w_{1}\left(1-w_{0}^{*} w_{0}\right)\right)+\sum_{j=2}^{n} \lambda_{j} T_{w_{0}}\left(w_{j}\right)=(\text { by }(c))=\sum_{j=1}^{n} \lambda_{j} f\left(w_{j}\right) .
\end{gathered}
$$

(e) Since elements in $\left(1-v v^{*}\right) B(K)\left(1-v^{*} v\right)$ can be approximated in norm by finite real linear combinations of mutually orthogonal partial isometries in $(1-$ $\left.v v^{*}\right) B(K)\left(1-v^{*} v\right)$ (see [20, Lemma 3.11]), and $f$ and $T_{v}$ are isometries, we derive from $(c)$ and $(d)$ that $f(x)=T_{v}(x)$ for every $x \in S\left(\mathcal{B}_{\left(1-v v^{*}\right) B(K)\left(1-v^{*} v\right)}\right)$.

$(f)$ Let $w$ be a partial isometry in $B(K)$. As before, let $w_{0}$ be a minimal partial isometry in $B(K)$ satisfying $w=w_{0}+w_{0}^{\perp}$, with $w_{0}^{\perp}=\left(1-w_{0} w_{0}^{*}\right) w\left(1-w_{0}^{*} w_{0}\right)$. Having in mind that $T_{w_{0}}$ is a surjective real linear isometry between spaces isometrically isomorphic to $B\left(\left(1-w_{0} w_{0}^{*}\right)(K)\right)$ and $B\left(\left(1-f\left(w_{0}\right) f\left(w_{0}\right)^{*}\right)(H)\right)$, Theorem 5.1 in [4] assures that $T_{w_{0}}$ preserves triple products of the form $\{a, b, c\}=\frac{1}{2}\left(a b^{*} c+c b^{*} a\right)$, and thus $T_{w_{0}}\left(w_{0}^{\perp}\right)$ is a partial isometry. By Theorem 2.5, we get

$$
f(w)=f\left(w_{0}+w_{0}^{\perp}\right)=f\left(w_{0}\right)+T_{w_{0}}\left(w_{0}^{\perp}\right)
$$


is the sum of two orthogonal partial isometries in $B(H)$, and then $f(w)$ is a partial isometry.

(g) Suppose $v_{1}, v_{2}$ are mutually orthogonal partial isometries in $B(K)$. Let us pick a non-zero $x \in\left(1-v_{1} v_{1}^{*}\right) B(K)\left(1-v_{1}^{*} v_{1}\right) \cap\left(1-v_{2} v_{2}^{*}\right) B(K)\left(1-v_{2}^{*} v_{2}\right)$. The equality $T_{v_{1}}\left(\frac{x}{\|x\|}\right)=f\left(\frac{x}{\|x\|}\right)=T_{v_{2}}\left(\frac{x}{\|x\|}\right)$ holds by $(e)$, and by linearity $T_{v_{1}}(x)=$ $T_{v_{2}}(x)$.

Finally statement $(h)$ follows straightforwardly from $(a)$ and $(g)$ because the dimensions of $H$ and $K$ are greater than or equal to 3 .

\section{SyNthesis OF A SURJECTIVE REAL LINEAR ISOMETRY}

In order to produce a real linear extension of our surjective isometry between $B(H)$ spaces, the next identity principle, which generalizes [25, Proposition 3.9], will play a central role.

Proposition 3.1. Let $H$ and $K$ be complex Hilbert spaces. Suppose that $f$ : $S(B(K)) \rightarrow S(B(H))$ is a surjective isometry, and $T: B(K) \rightarrow B(H)$ is a weak*continuous real linear operator such that $f(v)=T(v)$, for every minimal partial isometry $v$ in $B(K)$. Then $T$ and $f$ coincide on $S(B(K))$.

Proof. Take a minimal partial isometry $e$ in $B(K)$. By Theorem 2.7 $(b)$ and the hypothesis $T_{e}(v)=f(v)=T(v)$, for every minimal partial isometry $v$ in (1$\left.e e^{*}\right) B(K)\left(1-e^{*} e\right)$. Finite real linear combinations of mutually orthogonal minimal partial isometries in $\left(1-e e^{*}\right) B(K)\left(1-e^{*} e\right)$ are weak ${ }^{*}$-dense in $B(K)$, we therefore deduce from the weak ${ }^{*}$-continuity of $T_{e}$ and $T$ that $T_{e}=T$ on $\left(1-e e^{*}\right) B(K)(1-$ $\left.e^{*} e\right)$.

Pick a non-zero partial isometry $w$ in $B(K)$, and a minimal partial isometry $w_{0}$ such that $w=w_{0}+\left(1-w_{0} w_{0}^{*}\right) w\left(1-w_{0}^{*} w_{0}\right)$. By Theorem 2.5, the hypothesis and what we have proved in the first paragraph we obtain

$$
\begin{gathered}
f(w)=f\left(w_{0}\right)+T_{w_{0}}\left(\left(1-w_{0} w_{0}^{*}\right) w\left(1-w_{0}^{*} w_{0}\right)\right) \\
=T\left(w_{0}\right)+T\left(\left(1-w_{0} w_{0}^{*}\right) w\left(1-w_{0}^{*} w_{0}\right)\right)=T(w) .
\end{gathered}
$$

We have thus established that $T(w)=f(w)$ for every partial isometry $w$ in $B(K)$. Repeating the arguments in the proof of Theorem 2.7 $(e)$ we conclude that $T$ and $f$ coincide on $S(B(K))$.

We have developed enough tools to prove our main result.

Theorem 3.2. Let $H$ and $K$ be complex Hilbert spaces. Suppose that $f: S(B(K))$ $\rightarrow S(B(H))$ is a surjective isometry. Then there exists a surjective complex linear or conjugate linear surjective isometry $T: B(K) \rightarrow B(H)$ satisfying $f(x)=T(x)$, for every $x \in S(B(K))$.

Proof. By Riesz's lemma $H$ is finite dimensional if and only if $K$ is. When $H$ and $K$ are finite dimensional, the desired conclusion follows from [33] or [34.

We assume now that $H$ and $K$ are infinite dimensional. We shall apply the technique in [25, Theorem 3.13] to define our real linear isometry. Let $p_{1}, p_{2}$, and $p_{3}$ be three minimal projections in $B(K)$. Given $j \in\{1,2,3\}$, let $T_{p_{j}}:(1-$ $\left.p_{j}\right) B(K)\left(1-p_{j}\right) \rightarrow\left(1-f\left(p_{j}\right) f\left(p_{j}\right)^{*}\right) B(H)\left(1-f\left(p_{j}\right)^{*} f\left(p_{j}\right)\right)$ denote the surjective real linear isometry given by Theorem 2.5 . 
By Theorem 2.7 $(b)$ and $(h)$, the operators $T_{p_{1}}, T_{p_{2}}$, and $T_{p_{3}}$ are weak*-continuous, and they are all complex linear or conjugate linear. We assume that we are in the first case (the second case produces a conjugate linear map).

We can mimic the construction done in [25, Theorem 3.13] with the appropriate adaptations via the stronger properties developed in Section [2, Clearly, $B(K)$ admits the following decomposition:

$$
\begin{aligned}
B(K)= & \mathbb{C} p_{1} \oplus p_{1} B(K) p_{2} \oplus p_{2} B(K) p_{1} \oplus p_{1} B(K)\left(1-p_{1}-p_{2}\right) \\
& \oplus\left(1-p_{1}-p_{2}\right) B(K) p_{1} \oplus\left(1-p_{1}\right) B(K)\left(1-p_{1}\right) .
\end{aligned}
$$

We define a mapping $T: B(K) \rightarrow B(H)$ given by

$$
\begin{gathered}
T(x)=T_{p_{3}}\left(p_{1} x p_{1}\right)+T_{p_{3}}\left(p_{1} x p_{2}+p_{2} x p_{1}\right)+T_{p_{2}}\left(p_{1} x\left(1-p_{1}-p_{2}\right)+\left(1-p_{1}-p_{2}\right) x p_{1}\right) \\
+T_{p_{1}}\left(\left(1-p_{1}\right) x\left(1-p_{1}\right)\right) .
\end{gathered}
$$

The mapping $T$ is well defined, complex linear, and weak*-continuous thanks to the uniqueness of the above decomposition and the linearity and weak*-continuity of the mappings $T_{p_{1}}, T_{p_{2}}$, and $T_{p_{3}}$ (compare Theorem 2.7 $(b)$ ).

We shall conclude the proof by applying Proposition 3.1. For this purpose we shall show that

$$
T(e)=f(e) \text { for every minimal partial isometry } v \text { in } B(K) \text {. }
$$

Let $e$ be a minimal partial isometry in $B(K)$. Since $\operatorname{dim}(K)=\infty$ there exists a minimal projection $p_{4}$ satisfying $p_{4} \perp p_{1}, p_{2}, p_{3}, e$. The relations of orthogonality imply that

$$
p_{1} e p_{1}, p_{1} e p_{2}+p_{2} e p_{1}, p_{1} e\left(1-p_{1}-p_{2}\right)+\left(1-p_{1}-p_{2}\right) e p_{1},\left(1-p_{1}\right) e\left(1-p_{1}\right) \perp p_{4} .
$$

Equivalently, the elements $p_{1} e p_{1}, p_{1} e p_{2}+p_{2} e p_{1}, p_{1} e\left(1-p_{1}-p_{2}\right)+\left(1-p_{1}-p_{2}\right) e p_{1}$, $\left(1-p_{1}\right) e\left(1-p_{1}\right)$ all belong to $\left(1-p_{4}\right) B(K)\left(1-p_{4}\right)$. By definition, Theorem $2.7(g)$, and the previous observation we get

$$
\begin{gathered}
T(e)=T_{p_{3}}\left(p_{1} e p_{1}\right)+T_{p_{3}}\left(p_{1} e p_{2}+p_{2} e p_{1}\right)+T_{p_{2}}\left(p_{1} e\left(1-p_{1}-p_{2}\right)+\left(1-p_{1}-p_{2}\right) e p_{1}\right) \\
+T_{p_{1}}\left(\left(1-p_{1}\right) e\left(1-p_{1}\right)\right)=T_{p_{4}}\left(p_{1} e p_{1}\right)+T_{p_{4}}\left(p_{1} e p_{2}+p_{2} e p_{1}\right) \\
+T_{p_{4}}\left(p_{1} e\left(1-p_{1}-p_{2}\right)+\left(1-p_{1}-p_{2}\right) e p_{1}\right)+T_{p_{4}}\left(\left(1-p_{1}\right) e\left(1-p_{1}\right)\right) \\
=T_{p_{4}}(e)=(\text { Theorem } 2.7(e))=f(e)
\end{gathered}
$$

which proves (9) and finishes the arguments.

We are now in position to extend Theorem 3.2 for $\ell_{\infty}$-sums of $B(H)$ spaces. In the proof presented here we revise the arguments in the proof of [25, Theorem 3.12] and we insert the appropriate modifications.

Since Proposition 2.1, Theorems 2.2 and 2.3 and Lemma 2.4 are valid for general Banach spaces and general $\mathrm{C}^{*}$-algebras, respectively, the proof of Theorem 2.5 can be literally applied to prove the following result.

Theorem 3.3. Let $\left(H_{i}\right)_{i \in I}$ be a family of complex Hilbert spaces, let $A$ be a $C^{*}$ algebra, and suppose that $f: S(A) \rightarrow S\left(\bigoplus_{i}^{\ell_{\infty}} B\left(H_{i}\right)\right)$ is a surjective isometry. Let e be a minimal partial isometry in $A$. Then $f(e)$ is a minimal partial isometry in $B=\bigoplus_{i}^{\ell_{\infty}} B\left(H_{i}\right)$, and there exists a surjective real linear isometry

$$
T_{e}:\left(1-e e^{*}\right) A\left(1-e^{*} e\right) \rightarrow\left(1-f(e) f(e)^{*}\right) B\left(1-f(e)^{*} f(e)\right)
$$


such that

$$
f(e+x)=f(e)+T_{e}(x) \text { for all } x \text { in } \mathcal{B}_{\left(1-e e^{*}\right) A\left(1-e^{*} e\right)} .
$$

In particular, the restriction of $f$ to the face $F_{e}=e+\left(1-e e^{*}\right) \mathcal{B}_{A}\left(1-e^{*} e\right)$ is a real affine function.

It should be noted here that statements $(a)$ and $(h)$ in Theorem 2.7 need not be true when $B(K)$ and $B(H)$ are replaced with von Neumann algebras of the form $\bigoplus_{j}^{\ell_{\infty}} B\left(K_{j}\right)$ and $\bigoplus_{i}^{\ell_{\infty}} B\left(H_{i}\right)$, respectively. However, this obstacle will be avoided in the proof of our last result with an appropriate version of Theorem 2.7.

Theorem 3.4. Let $\left(H_{i}\right)_{i \in I}$ and $\left(K_{j}\right)_{j \in J}$ be two families of complex Hilbert spaces. Suppose $f: S\left(\bigoplus_{j}^{\ell_{\infty}} B\left(K_{j}\right)\right) \rightarrow S\left(\bigoplus_{i}^{\ell_{\infty}} B\left(H_{i}\right)\right)$ is a surjective isometry. Then there exists a surjective real linear isometry $T: S\left(\bigoplus_{j}^{\ell_{\infty}} B\left(K_{j}\right)\right) \rightarrow S\left(\bigoplus_{i}^{\ell_{\infty}} B\left(H_{i}\right)\right)$ satisfying $\left.T\right|_{S(E)}=f$.

Proof. To simplify the notation, set $A=\bigoplus_{j}^{\ell_{\infty}} B\left(K_{j}\right)$ and $B=\bigoplus_{i}^{\ell_{\infty}} B\left(H_{i}\right)$. If $\sharp J \geq 2$, we can pick two different subindexes $j_{1}$ and $j_{2}$ in $J$. Let $p_{1} \in B\left(K_{j_{1}}\right)$ and $p_{2} \in B\left(K_{j_{2}}\right)$ be minimal projections, and for $k=1,2$ let

$$
T_{p_{k}}:\left(1-p_{j_{k}}\right) A\left(1-p_{j_{k}}\right) \rightarrow\left(1-f\left(p_{j_{k}}\right) f\left(p_{j_{k}}\right)^{*}\right) B\left(1-f\left(p_{j_{k}}\right)^{*} f\left(p_{j_{k}}\right)\right)
$$

be the surjective real linear isometry given by Theorem 3.3 .

Let us observe that we can write $A=A_{1} \oplus A_{2}$, where $A_{2}=\bigoplus_{j \neq j_{1}}^{\ell_{\infty}} B\left(K_{j}\right)$ and $A_{1}=B\left(K_{j_{1}}\right)$. The symbol $\pi_{i}$ will stand for the projection of $A$ onto $A_{i}$. The mapping $T: A \rightarrow B, T(x):=T_{p_{2}}\left(\pi_{1}(x)\right)+T_{p_{1}}\left(\pi_{2}(x)\right)$ is well defined, real linear and continuous. Lemma 2.13 in [16] proves that $T_{p_{1}}$ and $T_{p_{2}}$ (and hence $T$ ) are weak*-continuous.

We shall prove next that for each minimal partial isometry $v \in A$

$$
\text { the surjective real linear isometry } T_{v} \text { given by Theorem } 3.3
$$

$$
\text { preserves triple products of the form }\{a, b, c\}=2^{-1}\left(a b^{*} c+c b^{*} a\right) \text {. }
$$

Take a minimal partial isometry $v$ in $A$. We deduce from the structure of $A$ and the minimality of $v$ the existence of a unique index $j_{0}$ in $J$ such that $v$ is a minimal partial isometry in $B\left(K_{j_{0}}\right)$. Therefore,

$$
\left(1-v v^{*}\right) A\left(1-v^{*} v\right)=\bigoplus_{j \neq j_{0}}^{\ell_{\infty}} B\left(K_{j}\right) \oplus^{\infty}\left(\left(1-v v^{*}\right) B\left(K_{j_{0}}\right)\left(1-v^{*} v\right)\right),
$$

and precisely one of the next statements holds:

(i) $\left(1-v v^{*}\right) A\left(1-v^{*} v\right)=\bigoplus_{j \neq j_{0}}^{\ell_{\infty}} B\left(K_{j}\right)$ if $\operatorname{dim}\left(K_{j_{0}}\right)=1$;

(ii) $\left(1-v v^{*}\right) A\left(1-v^{*} v\right) \cong \bigoplus_{j \neq j_{0}}^{\ell_{\infty}} B\left(K_{j}\right) \oplus^{\infty} \mathbb{C}$ if $\operatorname{dim}\left(K_{j_{0}}\right)=2$;

(iii) $\left(1-v v^{*}\right) A\left(1-v^{*} v\right)=\bigoplus_{j \neq j_{0}}^{\ell_{\infty}} B\left(K_{j}\right) \oplus^{\infty} B\left(K_{j_{0}}^{1}, K_{j_{0}}^{2}\right)$ if $\operatorname{dim}\left(K_{j_{0}}\right) \geq 3$, where $K_{j_{0}}^{2}=\left(1-v v^{*}\right)\left(K_{j_{0}}\right)$ and $K_{j_{0}}^{1}=\left(1-v^{*} v\right)\left(K_{j_{0}}\right)$ are complex Hilbert spaces of dimension larger than or equal to 2 .

A similar decomposition holds for $\left(1-f(v) f(v)^{*}\right) B\left(1-f(v)^{*} f(v)\right)$. Arguing as in the proof of [5. Theorem 3.1], we can deduce, via [5, Propositions 1.1 and 2.6], that $T_{v}$ preserves triple products. In particular, for each $k=1,2$ the surjective real linear isometry $T_{p_{k}}$ preserves triple products. Therefore, $T_{v}$ preserves (minimal) 
partial isometries and orthogonality among elements in their respective domains (because, by [19, page 18], $a \perp b$ in $A$ if and only if $\{a, a, b\}=0$ ).

Let $K(A)=\bigoplus_{j}^{c_{0}} K\left(K_{j}\right)$, and $K(B)=\bigoplus_{i}^{c_{0}} K\left(H_{i}\right)$, where for a complex Hilbert space $H, K(H)$ denotes the $\mathrm{C}^{*}$-algebra of all compact operators on $H$. Clearly, $K(A)$ and $K(B)$ are compact $\mathrm{C}^{*}$-algebras. Let $v$ be an arbitrary minimal partial isometry in $A$. Theorem 3.3 assures that $f(v)$ and $f(-v)$ are minimal partial isometries in $B$. So, $f(v)$ and $f(-v)$ belong to $K(B)$. By hypothesis, we have $\|f(v)-f(-v)\|=\|v+v\|=2$, and thus Lemma 3.5 in 25] implies that

$$
f(-v)=-f(v)+\left(1-f(v) f(v)^{*}\right) K(B)\left(1-f(v)^{*} f(v)\right),
$$

which combined with the minimality of $f(-v)$ proves that

$$
f(-v)=-f(v) \text { for every minimal partial isometry } v \in A .
$$

We claim that

$$
T(v)=f(v) \text { for every minimal partial isometry } v \in A .
$$

Indeed, every minimal partial isometry $v$ in $A$ lies in $A_{1}$ or in $A_{2}$. If $v \in A_{1}$ (respectively, in $A_{2}$ ) we have $T(v)=T_{p_{2}}(v)$ (respectively, $T(v)=T_{p_{1}}(v)$ ) by definition.

Suppose first that $v \in A_{2}$. By Theorem 3.3 and (11) we know that $f(v)$ and $T(v)=T_{p_{1}}(v)$ are minimal partial isometries in $B$. Let $T_{v}$ denote the surjective real linear isometry given by Theorem 3.3 . We know from the just quoted theorem and (12) that

$$
f(v)+T_{v}\left(p_{1}\right)=f\left(p_{1}+v\right)=f\left(p_{1}\right)+T_{p_{1}}(v)=f\left(p_{1}\right)+T(v)
$$

and

$f(v)-T_{v}\left(p_{1}\right)=f(v)+T_{v}\left(-p_{1}\right)=f\left(-p_{1}+v\right)=f\left(-p_{1}\right)+T_{-p_{1}}(v)=-f\left(p_{1}\right)+T_{-p_{1}}(v)$.

Adding the last two identities we get $2 f(v)=T(v)+T_{-p_{1}}(v)$. Since $f(v), T(v)$, and $T_{-p_{1}}(v)$ are minimal partial isometries with $\left\|T(v)+T_{-p_{1}}(v)\right\|=\|2 f(v)\|=2$, a new application of [25. Lemma 3.5] implies that $T(v)=T_{-p_{1}}(v)$, and thus $f(v)=$ $T(v)=T_{-p_{1}}(v)=T_{p_{1}}(v)$, as claimed.

If $v \in A_{1}$, similar arguments can be applied to show that

$$
f(v)+T_{v}\left(p_{2}\right)=f\left(p_{2}+v\right)=f\left(p_{2}\right)+T_{p_{2}}(v)=f\left(p_{2}\right)+T(v)
$$

and

$f(v)-T_{v}\left(p_{2}\right)=f(v)+T_{v}\left(-p_{2}\right)=f\left(-p_{2}+v\right)=f\left(-p_{2}\right)+T_{-p_{2}}(v)=-f\left(p_{2}\right)+T_{-p_{2}}(v)$, which implies that $2 f(v)=T(v)+T_{-p_{2}}(v)$. Since $f(v), T(v)=T_{p_{2}}(v)$, and $T_{-p_{2}}(v)$ are minimal partial isometries with $\left\|T(v)+T_{-p_{2}}(v)\right\|=\|2 f(v)\|=2$, it follows from [25. Lemma 3.5] that $T(v)=T_{-p_{2}}(v)$, and $f(v)=T(v)=T_{-p_{2}}(v)=T_{p_{2}}(v)$. This finishes the proof of the claim in (13).

On the other hand, let $v$ and $w$ be minimal partial isometries in $A$ with $v \perp w$. The elements $T_{w}(v)$ and $T_{v}(w)$ are minimal partial isometries (compare (11)), and we deduce from Theorem 3.3 , (12), and (13) that

$\pm T(w)+T_{ \pm w}(v)= \pm f(w)+T_{ \pm w}(v)=f(v \pm w)=f(v) \pm T_{v}(w)=T(v) \pm T_{v}(w)$, which implies that $T_{w}(v)+T_{-w}(v)=2 T(v)=2 f(v)$ (that is, $\left\|T_{w}(v)+T_{-w}(v)\right\|=2$ ), and then $T_{w}(v)=T_{-w}(v)=T(v)$ (just apply [25, Lemma 3.5]).

We have shown that for each minimal partial isometry $v$ in $A$ we have $T(v)=$ $f(v)$. Furthermore, if $w$ is another minimal partial isometry with $v \perp w$, then $T_{w}(v)=T(v)$. Let $e$ be an arbitrary partial isometry in $A$, and let us find a 
minimal partial isometry $v \in A$ such that $e=v+\left(1-v v^{*}\right) e\left(1-v^{*} v\right)$. It is known that $e_{0}=\left(1-v v^{*}\right) e\left(1-v^{*} v\right)=w^{*}-\sum_{\lambda} v_{\lambda}$, where $\left\{v_{\lambda}\right\}_{\lambda}$ is a family of mutually orthogonal minimal partial isometries in $A$ with $v \perp v_{\lambda}$ for every $\lambda$. By Theorem 3.3 and the weak ${ }^{*}$-continuity of $T$ and $T_{v}$ we have

$$
\begin{aligned}
& f(e)=f(v)+T_{v}\left(e_{0}\right)=f(v)+w^{*}-\sum_{\lambda} T_{v}\left(v_{\lambda}\right) \\
& =T(v)+w^{*}-\sum_{\lambda} T\left(v_{\lambda}\right)=T(v)+T\left(e_{0}\right)=T(e) .
\end{aligned}
$$

That is, $f(e)=T(e)$ for every partial isometry $e$ in $A$. We have further shown that $T(e)=T_{v}(e)$ for every pair of partial isometries $e, v$ in $A$ with $v \perp e$ and $v$ minimal.

We can now argue as in the proof of Theorem 2.7 $(d)$ to show that

$$
f\left(\sum_{j=1}^{n} \lambda_{j} w_{j}\right)=\sum_{j=1}^{n} \lambda_{j} f\left(w_{j}\right)=T\left(\sum_{j=1}^{n} \lambda_{j} w_{j}\right),
$$

for every set $\left\{w_{1}, \ldots, w_{n}\right\}$ of mutually orthogonal non-zero partial isometries in $A$, and $\lambda_{1}, \ldots, \lambda_{n}$ in $\mathbb{R}^{+}$with $\lambda_{1}=1$, and $\lambda_{j} \leq 1$ for all $j$. Indeed, pick a minimal partial isometry $v_{1}$ such that $w_{1}=v_{1}+w_{1}^{\perp}$, where $w_{1}^{\perp} \perp v_{1}$, by replacing $w_{1}$ with $v_{1}$ we can always assume that $w_{1}$ is minimal. By Theorem 3.3 and the above properties we have

$$
\begin{aligned}
& f\left(\sum_{j=1}^{n} \lambda_{j} w_{j}\right)=f\left(w_{1}\right)+T_{w_{1}}\left(\sum_{j=2}^{n} \lambda_{j} w_{j}\right)=f\left(w_{1}\right)+\sum_{j=2}^{n} \lambda_{j} T_{w_{1}}\left(w_{j}\right) \\
= & T\left(w_{1}\right)+\sum_{j=2}^{n} \lambda_{j} T\left(w_{j}\right)=\sum_{j=1}^{n} \lambda_{j} f\left(w_{j}\right)=\sum_{j=1}^{n} \lambda_{j} T\left(w_{j}\right)=T\left(\sum_{j=1}^{n} \lambda_{j} w_{j}\right) .
\end{aligned}
$$

Since every element in $S(A)$ can be approximated in norm by finite real linear combinations of mutually orthogonal partial isometries in $A$ (see [20, Lemma 3.11]), we derive from the above properties that $f(x)=T(x)$ for every $x \in S(A)$.

If $\sharp I \geq 2$ we can apply the above arguments to $f^{-1}$. Finally, if we assume that $\sharp J=\sharp I=1$, then the desired statement follows from Theorem 3.2 .

\section{ACKNOWLEDGMENTS}

The authors were partially supported by the Spanish Ministry of Economy and Competitiveness and European Regional Development Fund project no. MTM201458984-P and Junta de Andalucía grant FQM375.

The authors are very grateful to the anonymous referee for pointing out a gap in the original proof of Theorem 3.4 and some other valuable suggestions to improve the final version of this paper.

\section{REFERENCES}

[1] C. A. Akemann and G. K. Pedersen, Ideal perturbations of elements in $C^{*}$-algebras, Math. Scand. 41 (1977), no. 1, 117-139. MR0473848

[2] C. A. Akemann and G. K. Pedersen, Facial structure in operator algebra theory, Proc. London Math. Soc. (3) 64 (1992), no. 2, 418-448. MR.1143231 
[3] L. Cheng and Y. Dong, On a generalized Mazur-Ulam question: extension of isometries between unit spheres of Banach spaces, J. Math. Anal. Appl. 377 (2011), no. 2, 464-470. MR2769149

[4] C.-H. Chu, T. Dang, B. Russo, and B. Ventura, Surjective isometries of real $C^{*}$-algebras, J. London Math. Soc. (2) 47 (1993), no. 1, 97-118. MR.1200981

[5] T. Dang, Real isometries between JB*-triples, Proc. Amer. Math. Soc. 114 (1992), no. 4, 971-980. MR 1056677

[6] W. J. Davis, T. Figiel, W. B. Johnson, and A. Pełczyński, Factoring weakly compact operators, J. Functional Analysis 17 (1974), 311-327. MR0355536

[7] G. Ding, The 1-Lipschitz mapping between the unit spheres of two Hilbert spaces can be extended to a real linear isometry of the whole space, Sci. China Ser. A 45 (2002), no. 4, 479-483. MR 1912120

[8] G. Ding, The isometric extension problem in the unit spheres of $l^{p}(\Gamma)(p>1)$ type spaces, Sci. China Ser. A 46 (2003), no. 3, 333-338. MR2010142

[9] G. G. Ding, On the extension of isometries between unit spheres of $E$ and $C(\Omega)$, Acta Math. Sin. (Engl. Ser.) 19 (2003), no. 4, 793-800. MR2023371

[10] G. Ding, The representation theorem of onto isometric mappings between two unit spheres of $l^{\infty}$-type spaces and the application on isometric extension problem, Sci. China Ser. A 47 (2004), no. 5, 722-729. MR2127202

[11] G. G. Ding, The representation theorem of onto isometric mappings between two unit spheres of $l^{1}(\Gamma)$ type spaces and the application to the isometric extension problem, Acta Math. Sin. (Engl. Ser.) 20 (2004), no. 6, 1089-1094. MR2130374

[12] G. Ding, On isometric extension problem between two unit spheres, Sci. China Ser. A 52 (2009), no. 10, 2069-2083. MR2550266

[13] C. M. Edwards, F. J. Fernández-Polo, C. S. Hoskin, and A. M. Peralta, On the facial structure of the unit ball in a JB*-triple, J. Reine Angew. Math. 641 (2010), 123-144. MR2643927

[14] C. M. Edwards and G. T. Rüttimann, On the facial structure of the unit balls in a JBW*triple and its predual, J. London Math. Soc. (2) 38 (1988), no. 2, 317-332. MR966303

[15] C. M. Edwards and G. T. Rüttimann, Compact tripotents in bi-dual JB*-triples, Math. Proc. Cambridge Philos. Soc. 120 (1996), no. 1, 155-173. MR.1373355

[16] F. J. Fernández-Polo, J. Martínez, and A. M. Peralta, Surjective isometries between real JB*-triples, Math. Proc. Cambridge Philos. Soc. 137 (2004), no. 3, 703-723. MR2103925

[17] F. J. Fernández-Polo and A. M. Peralta, Low rank compact operators and Tingley's problem, preprint 2016. arXiv:1611.10218v1

[18] F. J. Fernández-Polo, J. J. Garcés, A. M. Peralta, and I. Villanueva, Tingley's problem for spaces of trace class operators, Linear Algebra Appl. 529 (2017), 294-323. MR.3659805

[19] L. A. Harris, Bounded symmetric homogeneous domains in infinite dimensional spaces, Proceedings on Infinite Dimensional Holomorphy (Internat. Conf., Univ. Kentucky, Lexington, Ky., 1973), Springer, Berlin, 1974, pp. 13-40. Lecture Notes in Math., Vol. 364. MR0407330

[20] G. Horn, Characterization of the predual and ideal structure of a $\mathrm{JBW}^{*}$-triple, Math. Scand. 61 (1987), no. 1, 117-133. MR929400

[21] V. Kadets and M. Martín, Extension of isometries between unit spheres of finite-dimensional polyhedral Banach spaces, J. Math. Anal. Appl. 396 (2012), no. 2, 441-447. MR2961236

[22] P. Mankiewicz, On extension of isometries in normed linear spaces (English, with Russian summary), Bull. Acad. Polon. Sci. Sér. Sci. Math. Astronom. Phys. 20 (1972), 367-371. MR.0312214

[23] G. K. Pedersen, Measure theory for $C^{*}$ algebras. II, Math. Scand. 22 (1968), 63-74. MR.0246138

[24] G. K. Pedersen, $C^{*}$-algebras and their automorphism groups, London Mathematical Society Monographs, vol. 14, Academic Press, Inc. [Harcourt Brace Jovanovich, Publishers], LondonNew York, 1979. MR.548006

[25] A. M. Peralta and R. Tanaka, A solution to Tingley's problem for isometries between the unit spheres of compact $C^{*}$-algebras and $J B^{*}$-triples, to appear in Sci. China Math. arXiv:1608.06327v1.

[26] I. Raeburn and A. M. Sinclair, The $C^{*}$-algebra generated by two projections, Math. Scand. 65 (1989), no. 2, 278-290. MR 1050869

[27] S. Sakai, $C^{*}$-algebras and $W^{*}$-algebras, Springer-Verlag, New York-Heidelberg, 1971. Ergebnisse der Mathematik und ihrer Grenzgebiete, Band 60. MR.0442701 
[28] M. Takesaki, Theory of operator algebras. I, Springer-Verlag, New York-Heidelberg, 1979. MR548728

[29] D.-N. Tan, Extension of isometries on unit sphere of $L^{\infty}$, Taiwanese J. Math. 15 (2011), no. 2, 819-827. MR2810183

[30] D.-N. Tan, On extension of isometries on the unit spheres of $L^{p}$-spaces for $0<p \leq 1$, Nonlinear Anal. 74 (2011), no. 18, 6981-6987. MR2833687

[31] D. N. Tan, Extension of isometries on the unit sphere of $L^{p}$ spaces, Acta Math. Sin. (Engl. Ser.) 28 (2012), no. 6, 1197-1208. MR2916333

[32] R. Tanaka, A further property of spherical isometries, Bull. Aust. Math. Soc. 90 (2014), no. 2, 304-310. MR3252013

[33] R. Tanaka, The solution of Tingley's problem for the operator norm unit sphere of complex $n \times n$ matrices, Linear Algebra Appl. 494 (2016), 274-285. MR.3455697

[34] R. Tanaka, Spherical isometries of finite dimensional $C^{*}$-algebras, J. Math. Anal. Appl. 445 (2017), no. 1, 337-341. MR.3543770

[35] R. Tanaka, Tingley's problem on finite von Neumann algebras, J. Math. Anal. Appl. 451 (2017), no. 1, 319-326. MR.3619239

[36] D. Tingley, Isometries of the unit sphere, Geom. Dedicata 22 (1987), no. 3, 371-378. MR.887583

[37] R. S. Wang, Isometries between the unit spheres of $C_{0}(\Omega)$ type spaces, Acta Math. Sci. (English Ed.) 14 (1994), no. 1, 82-89. MR.1280087

[38] T. M. Rassias and P. M. Pardalos (eds.), Mathematics without boundaries, Springer, New York, 2014. Surveys in pure mathematics. MR.3307648

Departamento de Análisis Matemático, Facultad de Ciencias, Universidad de GranADA, 18071 Granada, Spain

Email address: pacopolo@ugr.es

Departamento de Análisis Matemático, Facultad de Ciencias, Universidad de GranADA, 18071 Granada, Spain

Email address: aperalta@ugr.es 